\title{
A cognição social dos psicopatas: achados científicos recentes
}

\author{
Social cognition in psychopaths: \\ Recent scientific findings
}

\author{
Silvio José Lemos VASCONCELLOS \\ Roberta SALVADOR-SILVA ${ }^{2}$ \\ Fernanda de VARGAS \\ Fernanda Xavier HOFFMEISTER ${ }^{1}$ \\ Priscila Flores PRATES \\ Renan Meirelles da SILVA ${ }^{1}$
}

\begin{abstract}
Resumo
O psicopata apresenta traços na personalidade relacionados à ausência de remorso e uma maior dominância social, expressos muitas vezes como manipulação de outros indivíduos. Em estudos atuais sugere-se que psicopatas podem apresentar deficiências no processamento de estímulos emocionais em uma situação de interação social. Este estudo tem por objetivo realizar uma revisão teórica, não sistemática, discutindo pesquisas recentes sobre o tema. A partir da leitura, conclui-se que novas considerações revelam-se pertinentes, pois nem sempre o tipo de disfunção da cognição social dos psicopatas é explícito na literatura. Sugere-se que outros métodos para avaliar a capacidade dos psicopatas em identificar as emoções possam ser investigadas, além de identificar a medida das estratégias interpessoais. Em termos gerais, apresenta-se uma proposta de reflexão para um transtorno cuja compreensão etiológica deve ser biopsicossocial, contribuindo para assinalar novas direções nas pesquisas voltadas para a cognição social dos psicopatas.
\end{abstract}

Palavras-chave: Cognição social; Emoções; Personalidade; Personalidade antissocial; Relações interpessoais.

\begin{abstract}
Psychopaths have personality traits related to the failure to feel remorse and greater social dominance, which are often expressed in their manipulation of others. Current studies have suggested that psychopaths may have deficiencies in processing emotional stimuli in situations of social interaction. The objective of this study is to conduct a literature review and discuss recent research on this topic. Based on the studies reviewed, it can be said that new considerations are important since the type of social cognition impairment in psychopaths is not commonly addressed in the literature. It is suggested the use of different methods to evaluate the ability of psychopaths to identify emotions that can be

\footnotetext{
1 Universidade Federal de Santa Maria, Departamento de Psicologia, Programa de Pós-Graduação em Psicologia da Saúde. R. Floriano Peixoto, 1750, $3^{\circ}$ andar, Sala 313, 97015-372, Centro, Santa Maria, RS, Brasil. Correspondência para/Correspondence to: S.J.L. VASCONCELLOS.E-mail: <silviojlvasco@hotmail.com>.

2 Pontifícia Universidade Católica do Rio Grande do Sul, Faculdade de Psicologia, Programa de Pós-Graduação em Psicologia. Porto Alegre, RS, Brasil
} 
investigated in addition to identifying the measure of interpersonal strategies. Overall, the present study proposes a reflection upon a disorder whose etiology should be understood from a biopsychosocial perspective, contributing to new directions in research on social cognition in psychopaths.

Keywords: Social cognition; Emotions; Personality; Antisocial personality; Interpersonal relations.

A psicopatia pode ser entendida como um conjunto de traços de personalidade relacionados à ausência de remorso, baixa empatia, impulsividade, busca por estimulação, além de uma maior dominância social, cuja expressão pode se dar a partir da capacidade de manipular outros indivíduos (Hauck Filho, Teixeira, \& Dias, 2012; Patrick, Fowles, \& Krueger, 2009). Características desse tipo já foram, em outras épocas, consideradas por proponentes de construtos que se assemelham ao atual conceito de psicopatia, a exemplo de denominações como loucura moral ou mania sem delírio (Pinel, 2007). Essa última denominação, conforme Pinel, descreve indivíduos que não apresentariam lesões intelectuais, mas apenas afetivas, apresentando ainda um furor instintivo e uma maior propensão à violência (Pinel, 2007). Aproximações conceituais dessa natureza, bem como considerações sobre o trabalho de autores mais recentes que se apoiam no uso das escalas que avaliam o transtorno, permitem considerar que a própria capacidade de manipulação no contexto interpessoal pode estar no cerne do quadro.

Estudos atuais, no entanto, sugerem que psicopatas também podem apresentar deficiências específicas no que se refere ao processamento de estímulos emocionais em uma situação de interação social. Deficits na chamada cognição social que, por sua vez, abarca a capacidade de processar e ponderar informações sociais podem, nesse sentido, estar presentes como um elemento constitutivo do quadro (Flavell, Miller, \& Miller, 1999). Uma menor capacidade para identificar expressões faciais que explicitam emoções negativas tem sido, por exemplo, verificada em psicopatas quando comparados a grupo controle (Sharp \& Vanwoerden, 2014; Vasconcellos, Salvador-Silva, Dias, Davoglio, \& Gauer, 2014a). Achados indicando um processamento deficitário em indivíduos que ainda não apresentam o transtorno plenamente consolidado também têm sido verificados em maior ou menor grau na literatura científica sobre o tema (Blair, Colledge, Murray, \& Mitchell, 2001).

Questionamentos sobre a coexistência desses mecanismos supostamente antagônicos mostram-se pertinentes para compreender a etiologia da psicopatia. Afinal, revela-se cabível perguntar como psicopatas podem ser, na maioria dos casos, manipuladores eficazes, sendo, ao mesmo tempo, processadores menos perspicazes no que diz respeito a determinadas informações sociais de conteúdo emocional. A explicação para esses achados supostamente paradoxais demanda uma análise aprofundada, bem como uma discussão embasada quanto às pesquisas recentes sobre o tema.

Dessa forma, entende-se que um artigo teórico pode viabilizar avanços sobre o entendimento desse construto, bem como subsidiar novas pesquisas nesse campo. Os autores do presente trabalho postulam, nesse sentido, que uma discussão teórica dessa natureza revela-se adequada para elucidar pontos que podem, em um primeiro momento, parecer obscuros ou mesmo cindidos quanto ao estado atual de conhecimento sobre o assunto. Faz-se necessário considerar, em termos científicos, esse aparente paradoxo. Entende-se ainda que explanações desse tipo revelam-se compatíveis com um artigo teórico sobre o tema, reservando um maior espaço para explicar o porquê da capacidade manipulativa dos psicopatas coexistir com determinadas deficiências de processamento. O presente trabalho visa, portanto, complementar estudos anteriores e gerar avanços nessa área.

Para tanto, foi realizado um levantamento das publicações feitas nos últimos dez anos e indexadas no portal Psyclnfo, por intermédio de uma busca feita com os descritores psychopathy e social cognition, bem como psychopathy e emotion. A partir disso, foram selecionados artigos abordando alterações no processamento de informações de conteúdo emocional em psicopatas, verificadas a 
partir de estudos experimentais envolvendo os processos psicológicos básicos. Essa revisão foi complementada a partir da busca de outros trabalhos voltados para o comportamento manipulativo associado ao transtorno e publicados no mesmo período.

\section{Aspectos etiológicos e neurocognitivos da psicopatia}

A psicopatia pode ser entendida como um transtorno cujas origens remetem a eventos neurobiológicos e psicossociais relativos ao desenvolvimento da personalidade. Isso significa dizer que as diferentes estruturas cerebrais verificadas como alteradas em psicopatas adultos revelam-se, no que se refere à suas regularidades funcionais, passíveis de manutenção ou modificação, conforme o próprio ambiente no qual o indivíduo se desenvolve (Perez, 2012). O considerável desenvolvimento volumétrico da amígdala cerebral até cerca de oito ou nove anos de idade, bem como o acréscimo de substância cinzenta que essa estrutura pode comportar em anos subsequentes (Uematsu et al., 2012), sugerem que fatores genéticos e ambientais podem interagir para a consolidação do transtorno na idade adulta (Fallon, 2006).

No que se refere às estruturas cerebrais envolvidas, dois modelos explicativos revelam-se promissores para uma compreensão do quadro, ainda que o estado atual de conhecimento sobre o assunto não permita inferir qual deles verdadeiramente retrata o número exato de áreas relacionadas, em termos causais, à psicopatia. Os modelos decorrentes dos trabalhos de Blair e Kiehl apregoam, indistintamente, que o sistema límbico, o qual comporta um conjunto de regiões cerebrais envolvidas na utilização da informação emocional, exerce uma significativa influência para os sintomas da psicopatia (Anderson \& Kiehl, 2012). O modelo de Blair, no entanto, possui maior ênfase nas disfunções amigdalares verificadas em psicopatas. A amígdala é conhecida como a "porta de entrada do sistema límbico" e permite formar associações entre as informações ambientais processadas e o próprio estado emocional do organismo (Ramachandran, 1998).
Já o Modelo da Disfunção Paralímbica proposto por Kiehl é mais abrangente e apoia-se nos pressupostos da citoarquitetura celular, mas não demonstra ser uma plena refutação do modelo anterior. Esse modelo considera fatores relativos à densidade, tipo e estrutura neuronal das regiões periféricas ao sistema límbico, bem como o papel das mesmas para o processamento de informações sociais (Kiehl, 2006). Nesses termos, estruturas como o giro temporal superior, cingulado anterior, cingulado posterior, córtex orbitofrontal, ínsula e regiões para-hipocampais são descritas como intervenientes nos sintomas afetivos, interpessoais e comportamentais da psicopatia (Ermer, Cope, Nyalakanti, Calhoun, \& Kiehl, 2012).

A recente ênfase verificada nos trabalhos de Blair, quanto ao papel do córtex ventromedial, estrutura contígua ao córtex orbitofrontal situada na extremidade inferior do lobo frontal e fortemente conectado à amígdala, também se encarrega de aproximar a compreensão neurocognitiva desses pesquisadores (Anderson \& Kiehl, 2012; Blair et al., 2001). Na atualidade, pressupõe-se que o córtex orbital como um todo, a partir da sua estreita interface com a ínsula, pode estar mais envolvido em mapear experiências agradáveis ou desagradáveis. Já o córtex ventromedial permitiria uma avaliação mais direta quanto ao fato de o indivíduo estar obtendo o que deseja ou evitando o que não deseja; porém a linha divisória entre essas estruturas pode ser difusa, um fato que faz com que alguns autores optem por usar apenas a palavra orbital para descrevê-las conjuntamente (Pinker, 2013).

No que se refere aos estudos de neuroimagem, dados concordantes com a disfuncionalidade amigdalar verificada em psicopatas, ampliam a possibilidade de que as diferentes facetas que perfazem o quadro possam ser mais bem compreendidas a partir de seus correlatos neurocognitivos. Um menor nível de ativação da amígdala tem sido verificado em psicopatas quando comparados a grupo controle diante da exposição de imagens de impacto emocional envolvendo a violação de normas morais (C.L. Harenski, Harenski, Shane, \& Kiehl, 2010), bem como para o processamento de emoções negativas (Dollan \& Fullam, 2009) e durante processo de condicionamento aversivo (Rilling et al., 2007). Altera- 
ções volumétricas, sugerindo um desenvolvimento atrofiado da amígdala também têm sido verificadas em psicopatas na literatura recente (Anderson \& Kiehl, 2012).

De outro modo, considerando, por exemplo, o papel multimodal dos núcleos constituintes da amígdala, Boccardi et al. (2011) demonstraram que alterações morfológicas dessa estrutura em psicopatas parecem não ocorrer em uma única e mesma direção. Dito de outro modo, o trabalho em questão permitiu a identificação de alguns núcleos que se apresentam diminuídos, ao mesmo tempo que outros estariam aumentados em psicopatas. Nesses termos, os núcleos basolaterais, fortemente conectados com o córtex orbitofrontal e que exercem um importante papel na memória emocional e uma função reforçadora basilar para a introjeção de valores sociais (Paré, 2003) apresentam-se diminuídos em psicopatas (Boccardi et al., 2011). Já os núcleos centrais e laterais, envolvidos no circuito de detecção de ameaças, revelaram-se aumentados na amostra avaliada (Boccardi et al., 2011).

Embora achados nesse campo se demonstrem incipientes e embora o estudo de Boccardi et al. (2011) não tenha controlado a questão da comorbidade com a dependência química, os dados sugerem que mecanismos diferentes podem, em termos de psicopatia, fundamentar-se na ocorrência concomitante de alterações neuroanatômicas e funcionais caracteristicamente distintas. Inferências desse tipo também podem ser estendidas para o modelo paralímbico de Kiehl, indicando a possibilidade de mecanismos compensatórios no que se refere à cognição social dos psicopatas, mas que, por certo, ainda demandam investigações mais aprofundadas.

\section{A capacidade dos psicopatas identificarem emoções expressas pela face}

Estudos recentes sugerem que os psicopatas apresentam uma capacidade diminuída quanto à identificação de determinadas emoções expressas pela face, quando comparados a grupo controle (Eisenbarth, Alpers, Segrè, Calogero, \& Angrilli, 2008; Iria \& Barbosa, 2009; Vasconcellos, Salvador-
-Silva, Gauer, \& Gauer, 2014b). De um modo geral, tais deficiências têm sido encontradas principalmente no que se refere ao processamento de emoções negativas. Nessa perspectiva, incluem-se estudos que evidenciaram deficits quanto à identificação da emoção de medo (Blair et al., 2004; Del Gaizo \& Falkenback, 2007; Iria \& Barbosa, 2009; Montagne et al., 2005; Vasconcellos et al., 2014b); medo e tristeza (Blair \& Coles, 2000; Blair et al., 2001; Stevens, Charman, \& Blair, 2001) e repugnância (Hansen, Johnsen, Waage, \& Thayer, 2008; Kosson, Suchy, Mayer, \& Libby, 2002).

Até o momento, constata-se que a maioria desses trabalhos vem utilizando o paradigma das seis emoções básicas e universais propostas nas pesquisas de Paul Ekman: medo, alegria, surpresa, raiva, repugnância e tristeza. Outras emoções, consideradas mais recentemente como fazendo parte do rol das emoções básicas, como a emoção de desprezo ou outras expressões capazes de traduzir emoções híbridas (mais de uma emoção sendo expressa ao mesmo tempo), não foram investigadas nos estudos sobre a capacidade de psicopatas identificarem emoções expressas pela face.

Variações quanto aos métodos utilizados, incluindo o controle do tempo de exposição dos estímulos (imagens das expressões faciais) e a intensidade das expressões, também têm sido, mais recentemente, verificadas em estudos nesse campo (Vasconcellos et al., 2014a). No entanto, a maioria dos achados indica que pode existir uma deficiência de processamento emocional em psicopatas relacionadas à própria capacidade de decodificar emoções expressas pela face. O refinamento metodológico em tais pesquisas sugere ainda que essa deficiência pode ser bastante sutil, sendo que a sua detecção irá depender da acurácia das tarefas elaboradas para esses mesmos fins (Vasconcellos et al., 2014a).

Constata-se, porém, que a simples existência de algum nível de deficiência, ainda que sutil e específico para determinadas emoções expressas pela face é, por si só, aparentemente pouco compatível com a capacidade de manipulação que costuma caracterizar os psicopatas. Explicar, portanto, uma possível razão de ser desses achados é 
a proposta do presente artigo, conforme ficará evidenciado na sequência deste trabalho. Ressalta-se, ainda, que essa mesma deficiência coaduna-se com estudos de neuroimagem anteriormente descritos, uma vez que o sistema límbico e, em termos ainda mais específicos, a amígdala, estão diretamente envolvidos nessa capacidade (Moul, Kilcross, \& Dadds, 2012).

\section{A manipulação e outras manifestações na esfera interpessoal}

Evidências sobre o fato de a psicopatia abarcar um comprometimento na esfera interpessoal têm sido obtidas principalmente por intermédio da Psicometria. Nesse sentido, uma série de estudos com as escalas Hare, destacando-se dentre os mesmos o Psychopath Checklist Revised (PCL-R; Hare, 2003), indicam que um dos fatores capazes de expressar um determinado traço latente do quadro envolve os sintomas interpessoais da psicopatia. Nesse mesmo instrumento, podem-se destacar, por exemplo, itens como manipulação, mentira patológica e charme superficial.

Outros testes, a exemplo da Medida Interpessoal de Psicopatia (IM-P, Interpersonal Measure of Psychopathy) (Kosson, Steuerwald, Forth, \& Kirkhart, 1997), também preconizam que psicopatas, em situações de interação social, tendem a valer-se de diferentes estratégias com o objetivo de persuadir e manipular seus interlocutores (Salvador-Silva, Vasconcellos, Davoglio, Gauer, \& Kosson, 2012). Nessa escala, itens relacionados à postura corporal, à entonação da voz, ao modo pelo qual o entrevistado se dirige ao entrevistador, ao contato por intermédio do olhar, à dramaticidade e à perseveração no tema, dentre outros, são considerados para efeito de pontuação (Davoglio, Gauer, Vasconcellos, \& Lühring, 2011). Tais manifestações estão, indubitavelmente, relacionadas a estratégias de manipulação que demandam um constante monitoramento relacionado à expressão das próprias emoções, bem como à identificação das emoções provocadas no entrevistador.

Observa-se, no entanto, que poucos estudos mensuraram diretamente desempenhos mais espe- cíficos de psicopatas, no que se refere às estratégias verbais e não verbais presentes em situações de interação social. Um estudo identificou que psicopatas tendem a acentuar estratégias interpessoais como forma de despistar a atenção do seu interlocutor diante das mentiras proferidas. Foi constatado, nesse grupo, maior movimento com a cabeça e fala mais rápida em situações de mentira deliberada (Klaver, Lee, \& Hart, 2007). Outro estudo utilizou uma ferramenta de análise linguística e identificou que psicopatas, quando comparados a grupo controle, incluem mais conjunções explicativas ao relatarem seus crimes, apresentam maior alusão às necessidades pessoais durante o relato, além de evidenciarem, de forma mais recorrente, disfluências e uso de tempos verbais no passado ao longo do discurso quando falam sobre atos antissociais (Hancock, Woodworth, \& Porter, 2013). Outro estudo analisou milhares de quadros constantes em filmagens nas quais foi possível observar 2437 expressões faciais. Utilizando um instrumento de autorrelato para avaliar traços de psicopatia, os resultados assinalaram que o fator diretamente associado à faceta interpessoal da psicopatia mostrou-se relacionado à brevidade da expressão facial não intencional e, por outro lado, que os psicopatas não estariam completamente imunes à possibilidade de vazamento emocional quando tentam ocultar ou despistar determinadas emoções expressas pela face (Porter, Brinke, Baker, \& Wallace, 2011).

Observa-se, dessa forma, que ainda são incipientes os estudos sobre as estratégias de manipulação de psicopatas em tempo real em situações de interação social. Conjuntamente, esses trabalhos indicam que as estratégias de manipulação empregadas no contexto interpessoal por parte de psicopatas atrelam-se a uma constante necessidade de avaliar as reações provocadas em seus interlocutores. Considerando o próprio fato de que a maior parte da comunicação humana se dá por uma via não verbal e dependente significativamente da identificação correta das expressões faciais alheias (Ekman, 2003), faz-se então necessário explicar a suposta incongruência dos achados descritos. Os autores apresentam, a partir disso, possíveis explicações para o aparente paradoxo. 


\section{Identificação e manipulação de estados emocionais}

Na linguagem rotineiramente verificada em veículos de divulgação científica ou da mídia em geral, é comum ler ou ouvir afirmações peremptórias sobre a impossibilidade dos psicopatas experimentarem determinadas emoções. Essa negativa ampla estende-se, em termos mais recentes, à divulgação de estudos que envolvem a própria identificação das emoções alheias em psicopatas. Porém, conforme já foi mencionado, tais achados sugerem apenas uma dificuldade dos psicopatas em reconhecerem emoções específicas.

O controle do tempo de exposição ou da intensidade desses estímulos - relacionados, por sua vez, às emoções básicas expressas pela face -, tem permitido ainda problematizar o quão sutil pode ser essa mesma dificuldade. Esse fato, por si só, atesta que qualquer dificuldade branda está longe de ser, em tais casos, sinônimo de uma incapacidade plena. Em estudos empíricos recentes, o controle do tempo de exposição tem gerado a possibilidade de comparar desempenhos específicos em tempos específicos no que se refere a psicopatas e grupo controle (Eisenbarth et al., 2008; Salvador-Silva, Grizon, \& Arteche, 2014; Vasconcellos et al., 2014a).

No trabalho de Eisenbarth et al. (2008), foi usada, por exemplo, exposição com tempo livre, bem como a partir de frames de 33 milissegundos. Esse é um tempo suficiente apenas para um enquadramento mínimo e detecção das características mais salientes da imagem, conforme sugerem estudos recentes (Potter, Wyble, Hagmann, \& McCourt, 2014). Já os trabalhos de Vasconcellos et al. (2014b) envolvendo a comparação entre adolescentes com e sem traços de psicopatia, e o trabalho de Salvador-Silva et al. (2014) comparando mulheres psicopatas, mulheres não psicopatas com transtorno da personalidade antissocial e grupo controle, valeram-se de tempos de 200 milissegundos, 500 milissegundos e 1 segundo para efeito dessas comparações.

Observou-se, nos dois últimos estudos cita156 dos, que, conforme se aumentava o tempo de expo- sição dos estímulos, também melhorava o desempenho dos psicopatas ou indivíduos com traços de psicopatia para a identificação das emoções negativas. Uma atenção especial pode ser dada à melhora vislumbrada na mudança de tempo de 200 milissegundos para 500 milissegundos. Uma sutil deficiência de processamento pode, de algum modo, ser parcialmente compensada quando a informação se torna mais disponível no que se refere ao seu uso em termos de memória visual de curto prazo em condições não patológicas (Sligte, Scholte, \& Lamme, 2008). Infere-se daí que o mesmo pode acontecer com a psicopatia no que se refere ao processamento de informações expressas pela face. Deve-se ressaltar, no entanto, que, considerando o caráter incipiente desses achados, a proposta do presente artigo é tão somente elucidar a direção dos mesmos e sinalizar algumas possibilidades para novos estudos que possam aprofundar as inferências aqui discutidas.

Por outro lado, destaca-se o fato de que a melhora no desempenho de psicopatas diante de um tempo brevemente mais dilatado de exposição das expressões faciais, não verificadas com a mesma intensidade no grupo controle, é condizente com as explicações neuropsicológicas já destacadas. Em outras palavras, as estruturas subcorticais que se mostram disfuncionais na psicopatia são exatamente aquelas que viabilizam um processamento mais rápido, porém não ponderado, das informações sociais (LeDoux, 1996). Assim, é possível que psicopatas, de um modo geral, viabilizem um modo compensatório de fazer uso dessas informações em um contexto de interação social. Tais deficiências não seriam sinônimo de insuficiências no que se refere aos mecanismos voltados para o uso de informações relativas aos estados mentais alheios. Cabe destacar, nesse sentido, que psicopatas apresentam desempenho satisfatório em tarefas voltadas para a teoria da mente, uma capacidade que contempla a possibilidade de compreender os estados mentais alheios (Richell et al., 2003).

Pressupõe-se, com base no estado atual de conhecimento sobre o assunto, que psicopatas apresentam deficits específicos, porém, bastante sutis em termos de cognição social. Esse mesmo grau de 
sutileza explicaria a concordância nem sempre presente em diferentes estudos que investigam a capacidade dos psicopatas identificarem emoções expressas pela face. De outro modo, explicaria ainda a possibilidade de que os sintomas diretamente relacionados a estratégias de manipulação no contexto interpessoal coexistam com tais deficiências de processamento.

De um modo geral, é possível que, em termos interpessoais, a manipulação eficaz não dependa de uma identificação incondicionalmente perspicaz no que se refere às emoções alheias. Pode existir um limiar de detecção das emoções a ser concebido como minimamente suficiente no uso das informações sociais em uma situação de interação. Até o presente momento, no entanto, os estudos nesse campo não permitem inferir qual seria esse limiar, ainda que alguns achados já sugiram que o tempo de 200 milissegundos é necessário e, em condições não patológicas (indivíduos saudáveis), suficiente para o processamento de uma expressão facial (Schyns, Petro, \& Smith, 2009).

$\mathrm{Na}$ condição de um estudo teórico, os autores, neste artigo, propuseram uma explanação sobre um elevado, porém nem sempre explícito, nível de convergência relacionado aos achados mais atuais sobre a cognição social dos psicopatas. Essa reflexão revela-se pertinente pelo próprio fato de que a etiologia da psicopatia não foi plenamente explicada. Na condição de um transtorno influenciado por fatores biológicos, psicológicos e sociais, faz-se necessário compreender a forma pela qual os indivíduos acometidos pelo mesmo conseguem atuar sobre as emoções e expectativas alheias.

Em outras palavras, o estado atual de conhecimento sobre o assunto permite inferir que a psicopatia abarca mecanismos aparentemente contrapostos. Entretanto, o nível em que os mesmos se apresentam demonstra ser a explicação quanto à possibilidade dessa coocorrência. Nesses termos, a possibilidade de estudar conjuntamente a capacidade de manipulação das emoções alheias em psicopatas e uma sutil deficiência no processamento de alguma dessas emoções parece ser um caminho promissor para as novas pesquisas, visando complementar uma série de investigações anteriores.

\section{Colaboradores}

Todos os autores contribuíram na concepção e desenho do estudo, análise de dados e redação final.

\section{Referências}

Anderson, N. E., \& Kiehl, K. A. (2012). The psychopath magnetized: Insights from brain imaging. Trends in Cognitive Science, 16(1), 52-60. http://dx.doi.org/10. 1016/j.tics.2011.11.008

Blair, R. J. R., \& Coles, M. (2000). Expression recognition and behavioral problems in early adolescence. Cognitive Development, 15(4), 421-434. http://dx. doi.org/10.1016/S0885-2014(01)00039-9

Blair, R. J. R., Colledge, E., Murray, L., \& Mitchell, D. G. (2001). A selective impairment in processing of sad and fearful expressions in children with psychopathic tendencies. Journal of Abnormal Child Psychology, 29(6), 491-498. http://dx.doi.org/10.1023/A:101222 5108281

Blair, R. J. R., Mitchell, D. G. V., Peschardt, K. S., Colledge, E., Leonard, R. A., Shine, J. H., Murray, J., \& Perrett, L. K. (2004). Reduced sensitivity to others' fearful expressions in psychopathic individuals. Personality and Individual Differences, 37(6), 1111-1122. http://dx.doi. org/10.1016/j.paid.2003.10.008

Boccardi, M., Frisoni, G. B., Hare, R. D., Cavedo, E., Najt, P., Pievani, M., ... Tiihonen J. (2011). Cortex and amygdala morphology in psychopathy. Psychiatry Research: Neuroimaging, 193(2), 85-92. http://dx.doi. org/10.1016/j.pscychresns.2010.12.013

Davoglio, T. R., Gauer, G. J. C., Vasconcellos, S. J. L., \& Lühring, G. (2011). Medida Interpessoal de Psicopatia (IM-P): estudo preliminar no contexto brasileiro. Trends in Psychiatry and Psychotherapy, 33(3), 147-155. http:// dx.doi.org/10.1590/S2237-60892011000300004

Del Gaizo, A. L., \& Falkenbach, D. M. (2007). Primary and secondary psychopathic-traits and their relationship to perception and experience of emotion. Personality and Individual Differences, 45(3), 206-212. http://dx. doi.org/10.1016/j.paid.2008.03.019

Dollan, M., \& Fullam, R. (2009). Psychopathy and functional magnetic resonance imaging blood oxygenation level-dependent responses to emotional faces in violent patients with schizophrenia. Biological Psychiatry, 66(6), 570-577. http://dx.doi.org/10.1016/j. biopsych.2009.03.019

Eisenbarth, H., Alpers, G. W., Segrè, D., Calogero, A., \& Angrilli, A. (2008). Perception and evaluation of emotional faces in women scoring high on psychopathy. Psychiatry Research, 159(1-2), 189-195. http://dx.doi. org/10.1016/j.psychres.2007.09.001 
Ekman, P. (2003). Emotions revealed: Recognizing faces and feelings to improve communication and emotional life. New York: Times Book.

Ermer, E., Cope, L. M., Nyalakanti, P. K., Calhoun, V. D., \& Kiehl, K. A. (2012). Aberrant paralimbic gray matter in criminal psychopathy. Journal of Abnormal and Social Psychology, 121(3), 649-658. http://dx.doi.org/ 10.1037/a0026371

Fallon, J. H. (2006). Neuroanatomical background to understanding the brain of the young psychopath. Ohio State Journal of Criminal Law, 3(2), 341-367. Retrived February 3, 2015, from http://moritzlaw. osu.edu/ osjcl/Articles/Volume3_2/Symposium/FallonPDF-03-29-06.pdf

Flavell, J. H., Miller, P., \& Miller, S. A. (1999). Desenvolvimento cognitivo. Porto Alegre: Artmed.

Hancock, J. T., Woodworth, M. T., \& Porter, S. (2013). Hungry like the wolf: A word-pattern analysis of the language of psychopaths. Legal and Criminological Psychology, 18(1), 102-114. http://dx.doi.org/10.11 11/j.2044-8333.2011.02025.x

Hansen, A. L., Johnsen, B. H., Waage, L., \& Thayer, J. F. (2008). Brief communication: Psychopathy and recognition of facial expression of emotion. Journal of Personality Disorders, 22(6), 639-645. http://dx. doi.org/10.1521/pedi.2008.22.6.639

Hare, R. D. (2003). The hare psychopathy checklist revised. Canada: Multi Health Systems.

Harenski, C. L., Harenski, K. A., Shane, M. S., \& Kiehl, K. A. (2010). Aberrant neural processing of moral violations in criminal psychopaths. Journal of Abnormal and Social Psychology, 119(4), 863-874. http://dx.doi.org/10.1037/a0020979

Hauck Filho, N., Teixeira, M. A. P., \& Dias, A. C. G. (2012). Psicopatia: uma perspectiva dimensional e não criminosa do constructo. Avances en Psicología Latinoamericana, 30(2), 317-327. Recuperado em fevereiro 3, 2015, de http://www.scielo.org.co/scielo. php?script=sci_arttext\&pid=S1794-472420120002 00008\&lng=pt\&nrm=iso\&tlng=pt

Iria, C., \& Barbosa, F. (2009). Perception of facial expressions of fear: Comparative research with criminal and non-criminal psychopaths. Journal of Forensic Psychiatry and Psychology, 20(1), 66-73. http://dx. doi.org/10.1080/14789940802214218

Kiehl, K. A. (2006). A cognitive neuroscience perspective on psychopathy: Evidence for paralimbic system dysfunction. Psychiatric Research, 142(2-3), 107-128. http://dx.doi.org/10.1016/j.psychres.2005.09.013

Klaver, J. R., Lee, Z., \& Hart, S. D. (2007). Psychopathy and nonverbal indicators of deception in offenders. Law and Human Behavior, 31(4), 337-351. http://dx. doi.org/10.1007/s10979-006-9063-7

Kosson, D. S., Steuerwald, B. L., Forth, A. E., \& Kirkhart, K. J. (1997). A new method for assessing the interpersonal behavior of psychopathic individuals: Preliminary validation studies. Psychological Assessment, 9(2), 89-101. http://dx.doi.org/10.1037/1040-3590. 9.2 .89

Kosson, D. S., Suchy, Y., Mayer, A. R., \& Libby, J. (2002). Facial affect recognition in criminal psychopaths. Emotion, 2(4), 398-411. http://dx.doi.org/10.1037// 1528-3542.2.4.398

LeDoux, J. (1996). The emotional brain. Nova York: Simon \& Schuster.

Montagne, B., Honka, J. V., Kesselsa, R. P. C., Frigerio, E., Burtd, M., Zandvoorta, M. J. E. V., ... Haan, E. H. F. (2005). Reduced efficiency in recognising fear in subjects scoring high on psychopathic personality characteristics. Personality and Individual and Differences, 38(1), 5-11. http://dx.doi.org/10.1016/j. paid.2004.02.008

Moul, C., Killcross, S., \& Dadds, M. R. (2012). A model of differential amygdala activation in psychopathy. Psychological Review, 119(4), 789-806. http://dx. doi.org/10.1037/a0029342

Paré, D. (2003). Role of basolateral amygdala in memory consolidation. Progress in Neurobiology, 70(5), 409-420. http://dx.doi.org/10.1016/S0301-0082(03)00104-7

Patrick, C. J., Fowles, D. C., \& Krueger, R. F. (2009). Triarchic conceptualization of psychopathy: Developmental origins of disinhibition, boldness, and meanness. Development and Psychopathology, 21(3), 913-938. http://dx.doi.org/10.1017/S0954579409 000492

Perez, P. R. (2012). The etiology of psychopathy: A neuropsychological perspective. Aggression and Violent Behavior, 17(6), 519-522. http://dx.doi.org/10. 1016/j.avb.2012.07.006

Pinel, P. (2007). Tratado médico-filosófico sobre a alienação mental ou mania. Porto Alegre: Universidade Federal do Rio Grande do Sul.

Pinker, S. (2013). Os bons anjos da nossa natureza: por que a violência diminuiu. São Paulo: Companhia das Letras.

Porter, S., Brinke, L. T., Baker, A., \& Wallace, B. (2011). Would I lie to you? "Leakage" in deceptive facial expressions relates to psychopathy and emotional intelligence. Personality and Individual Differences, 51(2), 133-137. http://dx.doi.org/10.1016/j.paid. 2011.03.031

Potter, M. C., Wyble, B., Hagmann, C. E., \& McCourt, E. S. (2014). Detecting meaning in RSVP at $13 \mathrm{~ms}$ per picture. Attention, Perception \& Psychophysics, 76(2), 270-279. http://dx.doi.org/10.3758/s13414-013-0 605-z

Ramachandran, V. S. (1998). Phantoms in the brain: Probing the mysteries of the human mind. New York: William Morrow. 
Richell, R. A., Mitchell, D. G., Newman, C., Leonard, A., Baron-Cohen, S., \& Blair, R. J. (2003). Theory of mind and psychopathy: Can psychopathic individuals read the 'language of the eyes'? Neuropsychologia, 41(5), 523-526. http://dx.doi.org/10.1016/S0028-3932 (02)00175-6

Rilling, J. K., Glenn, A. L., Jairam, M. R., Pagnoni, G., Goldsmith, D. R., Elfenbein, A. H., \& Lilienfeld, S. O. (2007). Neural correlates of social cooperation and non-cooperation as a function of psychopathy. Biological Psychiatry, 61(11), 1260-1271. http://dx.doi. org/10.1016/j.biopsych.2006.07.021

Salvador-Silva, R., Grizon, A., \& Arteche, A. X. (2014). Recognition of emotional faces in psychopathic women (Dissertação de mestrado não-publicada). Pontifícia Universidade Católica do Rio Grande do Sul. Recuperado em fevereiro 5, 2015 de http://www. researchgate.net/publication/264543798_ Recognition_of_Emotional_Baby_Faces_in_ Psychopathic_Women

Salvador-Silva, R., Vasconcellos, S. J. L., Davoglio, T., Gauer, G., \& Kosson, D. (2012). Psicopatia e comportamentos interpessoais em detentos: um estudo correlacional. Avaliação Psicológica, 11(2), 239-245. Recuperado em fevereiro 3, 2015, de http://pepsic.bvsalud.org/ scielo. php? pid=\$1677-04712012000200009\&script= sci_arttext

Schyns, P. G., Petro, L. S., \& Smith, M. L. (2009). Transmission of facial expressions of emotion coevolved with their efficient decoding in the brain: Behavioral and brain evidence. Plos One, 4(5), e5625. http://dx.doi.org/10.1371/journal.pone.0005625

Sharp, C., \& Vanwoerden, S. (2014). Social cognition: Empirical contribution. The developmental building blocks of psychopathic traits: Revisiting the role of theory of mind. Journal of Personality Disorders, 28(1), 78-95. http://dx.doi.org/10.1521/pedi.2014.28.1.78

Sligte, I. G., Scholte, H. S., \& Lamme, V. A. F. (2008). Are there multiple visual short-term memory stores? Plos One, 3(2), e1699. http://dx.doi.org/10.1371/journal. pone.0001699

Stevens, D., Charman, T., \& Blair, R. J. (2001). Recognition of emotion in facial expressions and vocal tones in children with psychopathic tendencies. Journal of Genetic Psychology, 162(2), 201-211. http://dx.doi. org/10.1080/00221320109597961

Uematsu, A., Matsui, M., Tanaka, C., Takahashi T., Noguchi, K., Suzuki, M., \& Nishijo, H. (2012). Developmental trajectories of amygdala and hippocampus from infancy to early adulthood in healthy individuals. Plos One, 7(10), e46970. http:// dx.doi.org/10.1371/journal.pone.0046970

Vasconcellos, S. J. L., Salvador-Silva, R., Dias, A. C., Davoglio, T., \& Gauer, G. (2014a). Psicopatia e reconhecimento de expressões faciais de emoções. Psicologia: Teoria e Pesquisa, 30(2), 125-134. http://dx.doi.org/ 10.1590/S0102-37722014000200001

Vasconcellos, S. J., Salvador-Silva, R., Gauer, V., \& Gauer, G. (2014b). Psychopathic traits in adolescents and recognition of emotion in facial expressions. Psicologia: Reflexão e Crítica, 27(4), 768-774. http://dx.doi.org/ 10.1590/1678-7153.201427417

Recebido: abril 29, 2014

Versão final: outubro 9, 2015

Aprovado: dezembro 3, 2015 
